\title{
Invariant Based Ship DC Power System Design
}

\author{
I. Kondratiev and R. Dougal \\ University of South Carolina \\ Columbia, USA \\ kondrati@engr.sc.edu
}

\begin{abstract}
This paper introduces an alternative to traditional design approach - a design which provides the system components with the rules of interaction (invariants) and uses computational resources already available in the controller to implement these rules. We obtain rules of in-system interaction by analyzing power sharing in $\mathbf{m}$-paralleled connected power sources feeding a complex load. We illustrate the design using the example of a system with an arbitrary number of paralleled buck converters feeding a constant power load. We introduce the found invariants into the system using synergetic control theory, analyze the closed loop performance, and define the stability conditions as well as conditions for compensating impact of constant power load, Finally, we exercise the design on a two-converter system, where we illustrate power sharing capabilities.
\end{abstract}

\section{INTRODUCTION}

DC power distribution is very attractive for ship autonomous power systems due to its simplified interface for power sources, higher energy efficiency and power density, and lower implementation and maintenance costs $[1,2]$. The success of DC distribution depends on a feasible solution for the system design, which, despite a wide range of load variation, can provide stable operation of the system, flexible power flow management and reconfigurability, as well as scalability and clarity of the design approach. A range of factors, including complexity of the system dynamics, interactions among power sources, and influence of constant power loads, make a search for a solution using simulation difficult, if not unreliable. In contrast, the shift of design focus from control set-points to the set of defined rules for the interaction of the elements within the system makes the synergetic control design process both more specific and feasible than the traditional approaches.

In traditional system design, when individual power sources are put together, the stability of the system is evaluated using source-load admittance space [3], and further power flow management is performed by providing the system components with current references defined using static power flow management or a dynamic power router [4]. In contrast, our design approach provides the system components with the rules of interaction (invariants) and use the computational resources already available in the controller to implement these rules. Such capabilities to introduce invariant manifolds into a closed loop system have been already demonstrated using synergetic control theory [5,6] and sliding mode control[7]. As it was reported in [8], the introduction of invariants in the closed system behavior reduces the dynamic order of the controlled system as well as chances of unstable behavior.

In this paper, we introduce the principle of design of complex dynamic power systems, where attractors and invariants set the rules and define the driving forces of the behavior of system components. This principle has been inspired by examples of similar behaviors in nature, i.e in complex ecological systems, one of which we use for illustration. As shown in Fig. 1, in order to capture an ant, an antlion larva creates a pit in dry sand. The cone surface of the pit represents a zero friction surface, i.e. an invariant, and the top of the cone, where the larva sits, is an attractor. Once an ant gets into the pit, the larva starts to throw sand from the bottom of the pit and creates a sliding motion in the pit walls. As a result, the ant slides along the pit wall (invariant) towards the larva (attractor). Such behavior of the antlion larva allows it to capture the ant with far less effort than running after it. In other words, maximum efficiency is achieved with minimized effort due to informed transformation of the environment based on the properties of the controlled element. We use similar principles in the design of a DC distribution system by providing the system with rules of interaction (invariants) among its components and by defining such conditions for the system components that they are forced to self-organize the implementation of these rules.

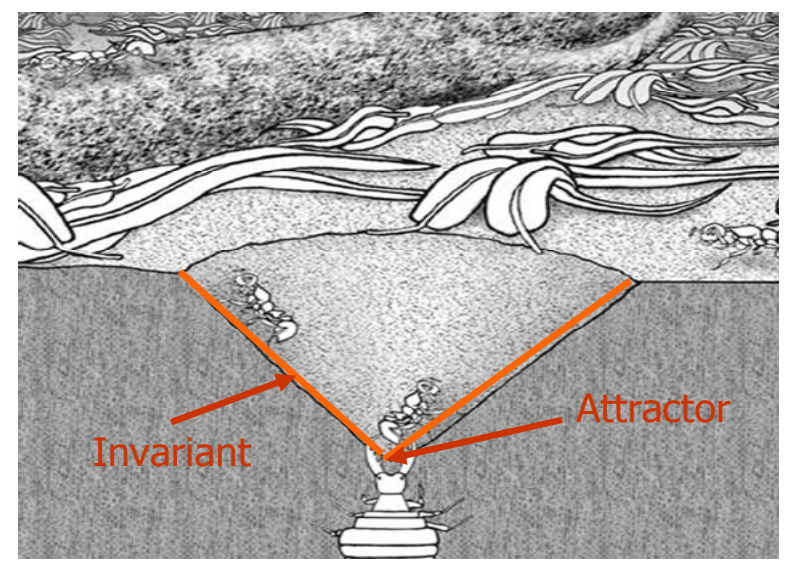

Fig 1. Antlion larva hunting for ants

We apply the synergetic control theory to introduce dynamic invariants suitable for use in ship DC distribution systems. We describe the benefits brought into the system under wide varying loading conditions by the suggested types of invariants, i.e. stable operation of the system, flexible power flow management and reconfigurability. We show that invariant based design of autonomous DC ship distribution power systems which relies on synergetic control theory provides a scalable design approach. As illustrated by the 
analysis of the system with an arbitrary number of paralleled converters feeding constant power load and by the simulation of two-converter sample systems, the invariant based design provides tools that can eliminate negative incremental impedance instability, flexibly reroute the power, and define the rules of dynamic power sharing during transients.

\section{INVARIANTS IN DC POWER SYSTEM}

Fig. 2 shows a simplified structure of a ship DC distribution system where sources $\mathrm{S}_{1}, . ., \mathrm{S}_{\mathrm{m}}$ represent converter based power sources connected in parallel and feeding one equivalent load (L) that substitutes service converter modules [2]. A source $\mathrm{S}_{\mathrm{m}+1}$ substitutes an uncontrolled capacitive energy storage connected directly to the system bus. A disturbance $M(t)$ represents a dependent current source, which allows us to take into account the change of the system load. Since in real life applications converter systems are usually influenced by sudden load change, the disturbance $M(t)$ is approximated by piece-wise constant function.

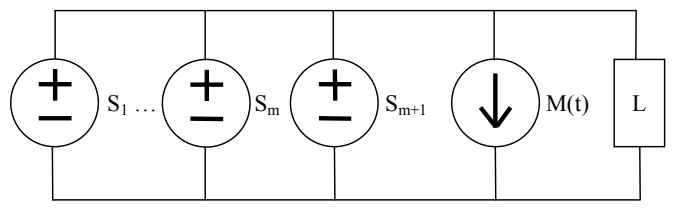

Fig.2 Simplified structure of a ship DC distribution system

Similar to [4], we define goals of power flow management as follows:

- $\quad$ Ensure predefined static power flow;

- Ensure predefined dynamic power flow;

- Provide power limitations to account for power source capability;

- Provide capability to account for a limited power production rate.

The power flow balance in the system is defined in (1).

$$
\sum_{i=1}^{m} P_{S i}+P_{S m+1}=P_{L}+v_{b} \cdot M(t)
$$

After substituting the expressions for power of each component of the system from (2), dividing the result by the bus voltage $v_{b}$, and grouping source currents $i_{s i}$ on the left side of the equation, we get expression (3). The equation (3) defines the power balance in the system during operation. However, in order to define power sharing among the sources, we need to define additional m-1 equations. Similarly to (4), we define these $\mathrm{m}-1$ equations as shown in (5).

$$
\begin{aligned}
& P_{S i}=i_{S i} \cdot v_{b} ; P_{S m+1}=\frac{d E_{C}}{d t}=C_{b} \cdot v_{b} \frac{d v_{b}}{d t} ; \\
& P_{L}=i_{L} \cdot v_{b}=\left(\frac{P_{L}}{v_{b}}+\frac{v_{b}}{R_{L}}\right) \cdot v_{b} \\
& \sum_{i=1}^{m} i_{S i}=\frac{P_{L}}{v_{b}}+\frac{v_{b}}{R_{L}}+M(t)-C_{b} \frac{d v_{b}}{d t} \\
& \sum_{i=1}^{m} i_{S i}=\frac{P_{L}}{v_{b}}+\frac{v_{b}}{R_{L}}+M(t)-C_{b} \cdot\left(v_{n}-v_{b}\right)
\end{aligned}
$$

$$
\sum_{i=1}^{m} a_{j i} \cdot i_{S i}=b_{j 1} \cdot M(t)+b_{j 2} \cdot v_{b}+b_{j 3} \cdot v_{n}+\frac{b_{j 4}}{v_{b}} \quad j=\overline{2, m}
$$

Joining equations (4) and (5) together, we obtain equations (6) that define the power flow in the system.

$$
\begin{aligned}
& A I=B_{1} X+B_{2} F_{3}(X) \\
& \text { where: } X=(M, v)^{T} \text { and } I=\left(i_{1}, \ldots, i_{m}\right)^{T} \\
& A=\left(\begin{array}{cccc}
a_{11} & a_{12} & \ldots & a_{1 m} \\
a_{21} & a_{22} & \ldots & a_{2 m} \\
\ldots & \ldots & \ldots & \ldots \\
a_{m 1} & a_{m 2} & \ldots & a_{m m}
\end{array}\right) \quad B_{1}=\left(\begin{array}{cc}
b_{11} & b_{12} \\
b_{21} & b_{22} \\
\ldots & \ldots \\
b_{m 1} & b_{m 2}
\end{array}\right) \\
& B_{2}=\left(\begin{array}{cc}
b_{13} & b_{14} \\
b_{23} & b_{24} \\
\ldots & \ldots \\
b_{m 3} & b_{m 4}
\end{array}\right) F_{3}(X)=\left(\begin{array}{ll}
v_{n} & \frac{1}{v_{b}}
\end{array}\right)^{T}
\end{aligned}
$$

The first equation in (6) represents the power conservation conditions in the system. The other m-1 equations in (6) define the power distribution among the power sources. Introduction of the saturation function in coefficients $a_{1 i}$ takes into account the power limitation imposed by the corresponding power source. A ramp-rate limitation imposed by the sources can be taken into account during control design by proper selection of corresponding time constant.

Invariants (6) introduced into the system via synergetic control will provide rules of behavior for the components of a closed loop system.

\section{Synergetic CONTROL THEORY}

Synergetic Control Theory [9, 10,11] exploits the capability of open systems to self-organize. The theory invokes a holistic philosophy of controlled dynamic interactions between energy, matter, and information which is implemented through a combination of both positive and negative feedback. The philosophy of synergetic control design is based on the principle of dynamic expansion and contraction of the state space of the controlled system. The expansion of the state space enriches the system dynamics by providing additional information that is the key to improving the performance of the closed-loop system. In contrast to expansion, the contraction of the state space that is performed by the control action eliminates the unwanted dynamics of the system or reduces excessive degrees of freedom. At the control design stage, these unwanted dynamics are removed by introducing dynamic constraints that are represented as invariant manifolds in the state space of the system.

To illustrate the concepts of the Synergetic Control Theory such as control design and assessment of stability, we use system (7) that is a scalar controllable nonlinear affine system representing an extended model of the initial system.

$$
\begin{aligned}
& \dot{x}_{i}=f_{i}(x) i=\overline{1, n-1} \\
& \dot{x}_{n}=f_{n}(x)+b_{n}(x) \cdot u
\end{aligned}
$$

where: $x=\left(x_{1}, x_{2}, \ldots, x_{n}\right)^{T}$ is a system state vector; $f_{i}$ are nonlinear smooth functions defining the dynamic evolution of the system; and $u$ is a control input. 
Synergetic Control Theory provides a method for generating control $u(x)=u(\psi)$ as a function of some specified macrovariables $\psi(x)$, which are called aggregated variables and which operate as order parameters of synergetic systems that define asymptotic properties of the system's motion [12].

For solving a control design problem, we define macrovariables $\psi(x)$ and use Kolesnikov's Functional (8) [13].

$$
J_{\Sigma}=\int_{0}^{\infty} F(\psi, \dot{\psi}) d t=\int_{0}^{\infty}\left(m^{2} \phi(\psi)+c^{2} \dot{\psi}\right) d t
$$

where: $F(\psi, \dot{\psi})$ is a continuous positive definite function; $\phi(\psi)$ is a smooth function that is chosen to be a) invertible and differentiable with respect to its arguments; b) $\phi(0)=0$; and c) $\phi(\psi)>0$ for all $\psi \neq 0 ; \psi(x)$ is either an aggregated variable or a macro-variable which defines an arbitrary, yet carefully chosen, either continuous or piece wise function of the state vector $\mathbf{x}$.

As was shown in [9], finding an optimal controller for the system (7) which minimizes functional (8) is equivalent to finding control (9) as a joint solution of the system (7) and of the so-called evolution equation (10).

$$
\begin{aligned}
& u=-b_{n}^{-1}(x) \frac{\partial \psi^{-1}}{\partial x_{n}}\left[\sum_{i=1}^{n} \frac{\partial \psi}{\partial x_{i}} f_{i}+\frac{1}{T} \phi(\psi)\right] \\
& \dot{\psi}(x)+\lambda \phi(\psi)=0
\end{aligned}
$$

Equation (10) defines the evolution of the system's macrovariable $\psi(x)$ into the invariant manifold (11).

$$
\psi(x)=0
$$

where: $\lambda=m / c=1 / T$; moreover from the synergetics point of view [12], $\lambda$ is a parameter proportional to the work performed by self-organizing forces in the system; it characterizes the speed of the self-organizing processes in the system. By choosing the value of $\lambda$, we can choose the desired speed of evolution of the self-organization.

Under control (9), manifold (11) becomes an attractor (or attracting set) in the state space of the closed-loop system. As a result, from an admissible arbitrary initial location in the state space, the system moves towards the manifold and then along the manifold to the equilibrium point.

The stability of the system's motion towards the manifold is determined by using Lyapunov function (12), a derivative of which (13) defines the basin of attraction of the attractor (11).

$$
\begin{aligned}
& V=0.5 \psi^{2}>0 \\
& \dot{V}(t)=-\lambda \phi(\psi)<0 \text { if } \lambda>0
\end{aligned}
$$

Equation (13) shows that the stability of the motion towards the attractor is not compromised by nonlinear properties of the system's model (7) and this motion is exponentially asymptotically stable, as long as the parameter $\lambda$ is greater than zero.

Once the system reaches the vicinity of the invariant manifold (11), the synergetic control law (9) will keep it on the manifold, thereby reducing the order of its dynamic behavior. The resulting model (14) is an exact decomposition of the dynamic system (7) that captures its slow dynamics. A reduced-order model (14) is obtained by substituting the manifold equation (11) into system (7) and replacing the equation with the control $u$.

$$
\begin{aligned}
& \dot{x}_{i}=f_{i}(x) i=\overline{1, n-1} \\
& \psi(x)=0
\end{aligned}
$$

In the vicinity of the manifold (11), the stability of the system motion can be analyzed using the first approximation of the model (14).

However, if we define macro-variable as $\psi=x_{n}-u_{1}$, the control design for system (14) can be recursively repeated assuming that now variable $x_{n}$ becomes an internal control $u_{1}$. The recursion can be continued until the specified order of the system dynamic is reached. The direct path of the recursion produces sequences of macro-variables $\psi_{i}(x)$, of optimized functionals (8), and of reduced order models (14). The return path of the recursion defines the control and the manifold structure. Recursive control design for a system with one control channel creates a sequential aggregation of invariant manifolds in the state space of the system and yields $a$ sequential optimization.

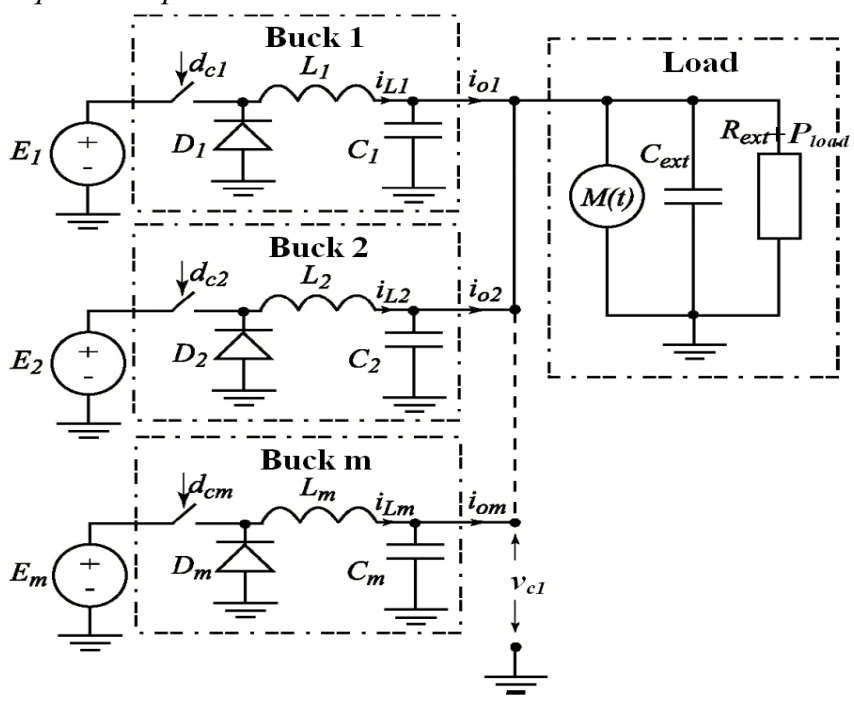

Fig. 3. System containing $\mathrm{m}$ parallel-connected buck converters

Without loss of generality, the same approach can be applied to systems having multiple control channels. In this case, the recursive design of synergetic controls results in sequences of macro-variables sets, of optimized functionals sets, and of reduced order models. The control obtained provides the system with a parallel-sequential aggregation of invariant manifolds and performs a multi-criteria optimization. The order of the dynamic motion along the intersection of these manifolds towards the equilibrium can be calculated using equation (15), where $m$ is the number of control channels and $l$ is the number of recursive steps.

$$
\operatorname{dim} x_{\psi}=n-m \cdot l
$$




\section{DESIGN IMPLEMENTATION}

To illustrate an invariant based control design, we use the system containing an arbitrary number of paralleled converters feeding constant power load that is presented in Fig.3.

\section{A. System model}

Assuming that the switching frequency of power converters is sufficiently fast, using similar assumptions as in [5], applying Kirchhoff's laws and averaging [14] to this system, the dynamics of the system can be described by(16).

The state space averaged model of the system presented in (16) is derived using the following assumptions: the system operates in a continuous conduction mode; state variables are the averaged $v_{c l}$ capacitor voltage and $i_{L i}$ inductance currents; constant power load is represented as a dependent current source the value of which equals to the power consumed by the load $\left(P_{\text {load }}\right)$ divided by the output voltage $\left(v_{c l}\right)$; a piece-wise constant current disturbance $M(t)$ sufficiently approximates variations of the system load and any variation of system parameters; the origin of the system model is at the specified steady state voltage; the zero order dynamics adequately represents the behavior of the sources $E_{i}$.

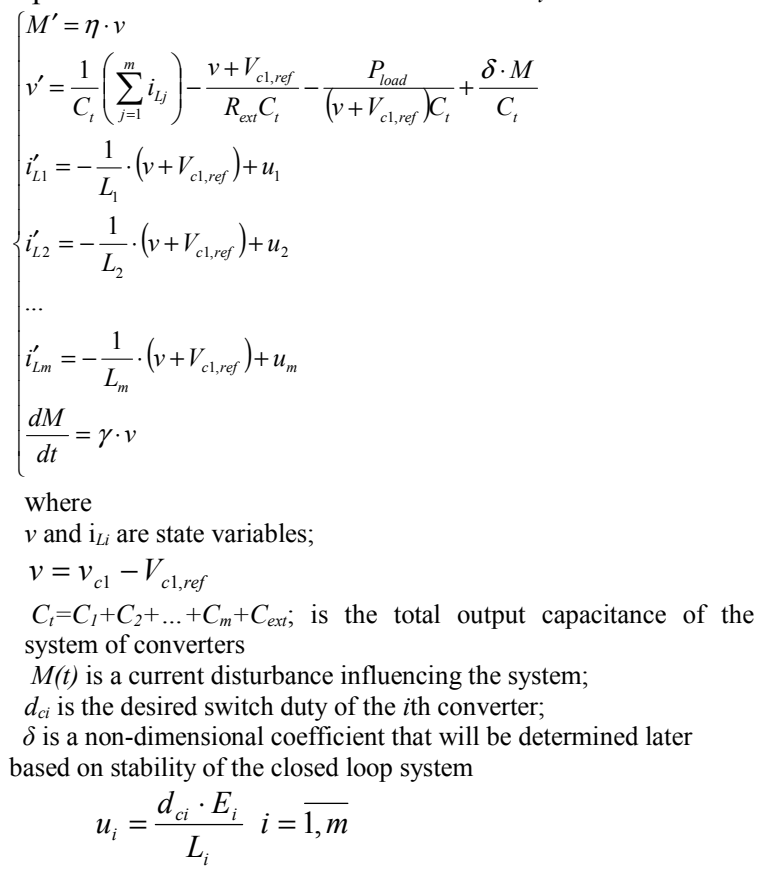

As a result of the introduction of an additional dynamic coordinate $M(t)$ into the system model (16), the system voltage becomes invariant to the system load resistance.

In order to simplify control derivation results, we represented system model (16) in the following form:

$\left\{\begin{array}{l}\dot{X}=F_{1} I+F_{2} X+C F_{3}(X) \\ \dot{I}=F_{4}(X, I)+U(X, I)\end{array}\right.$
where $X=(M, v)^{T}$ and $I=\left(i_{1}, \ldots, i_{m}\right)^{T}$

\section{B. Goals of the control design}

The primary goal of the control is to maintain a specified output voltage $V_{c l \text {,ref }}$, even while the system is affected by the disturbance $M(t)$. Another important goal is to ensure current sharing defined by (6). At the design stage, we do not make any particular assumptions about current sharing, except that the converter currents relate linearly to each other; that is, they, each, supply some constant fraction of the load current that corresponds to active current sharing. All these control design goals were incorporated into the system model by the corresponding coordinate transformation. The resulting control laws must ensure asymptotic stability of the closed loop system.

\section{Control synthesis procedure}

From a mathematical standpoint, synergetic control design [15] is based on a new method for generating control laws $u\left(\psi_{i}\right)=u_{i}(\mathrm{X}, \mathrm{I})$, or feedback, that direct the system from arbitrary initial conditions into the vicinity of the manifolds (18) and then ensure asymptotically stable motion along these manifolds toward the end attractors. On these attractors, the desired properties of the controlled system are guarantied. In short, the synergetic control design procedure is as follows. For system(17), the designer's specifications are formulated as a set of macro-variables (19) based on the system state variables. The number of macro-variables in the set equals the number of control channels.

$\psi_{i}(\mathrm{X}, \mathrm{I})=0$

$\psi_{i}=\psi_{i}(\mathrm{X}, \mathrm{I}), \mathrm{i}=\overline{1, m}$

Assuming that the desired evolution of the macro-variables is as in (20), substitution of the system model (17) in functional equation (20) results in an asymptotically stable control, which ensures the desired dynamic properties.

$T \cdot \dot{\Psi}+\Psi=0$

$T=E \cdot\left(\begin{array}{lll}T_{1} & \ldots & T_{m}\end{array}\right)^{T}$

$E$ - is unity matrix dimension $m x m$

Defining a macro-variable as shown in (21) and solving the system of functional equations (20) accounting for system model (17), yields control laws presented in (22).

$$
\begin{aligned}
& \Psi=A I-B_{1} X-B_{2} F_{3}(X) \\
& U(X, I)=A^{-1} \cdot\left(\left(B_{1}+B_{2} F_{4}\right)\left(F_{1} I+F_{2} X+C F_{3}(X)\right)-T^{-1} \Psi\right)+ \\
& -F_{4}(X, I)
\end{aligned}
$$

where:

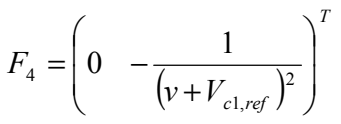

According to synergetic control theory [15], control laws (22) ensure asymptotically stable convergence towards the specified manifolds (18) within (3-5) $T_{i}$ and, as shown later, result in current sharing specified by coefficients in (21). 
D. Behavior on manifolds and stability of the closed-loop system

Under synergetic control, the closed loop system has an attracting set, which is the intersection of manifolds and which is reached by the representing point of the system. The study of the system behavior on the intersection of manifolds helps to understand the system stability. The properties of the system motion on manifolds are defined by the following system of algebraic and dynamic equations:

$$
\begin{aligned}
& \left\{\begin{array}{l}
\dot{X}=F_{1} I+F_{2} X+C F_{3}(X) \text { or } \\
\Psi=0
\end{array}\right. \\
& \left\{\begin{array}{l}
\dot{X}=F_{1} I+F_{2} X+C F_{3}(X) \\
I=-A^{-1}\left(B_{1} X+B_{2} F_{3}(X)\right)
\end{array}\right.
\end{aligned}
$$

In order to define the properties of the system dynamics on manifolds, we eliminate dependent variables, such as inductor currents, and derive a dynamic system (26).

$$
\dot{X}=\left(F_{2}-F_{1} A^{-1} B_{1}\right) X+\left(C-F_{1} A^{-1} B_{2}\right) F_{3}(X)
$$

The equation (26) shows that the behavior of the closed loop system on manifolds is defined by two components: linear and nonlinear. It also can be seen that manifold coefficients provide flexibility in the definition of both components. So, we can define matrix $B_{2}$ in such a way that the following conditions are true.

$C-F_{1} A^{-1} B_{2}=0$

Equation (27) represents the condition for counterweighing the nonlinear impact of a constant power load and for the linearization of the system (17). If these conditions are met, the equation (26) can be rewritten in the following form:

$$
\dot{X}=\left(F_{2}-F_{1} A^{-1} B_{1}\right) X
$$

The equation (28) defines a linear dynamic system, the properties of which depend on the choice of coefficients of matrix $A$ and $B_{1}$. The stability condition for the linearized system (28) can be defined by any linear technique.

Hence, synergetic control design results in analytical control laws that ensure asymptotic stability of the closed loop system and compensates for system nonlinearity by a particular choice of coefficients. The allocation of type and ratings of current sharing in the system is defined by coefficients of macro variables (6).

\section{Simulation Results}

In this section we present a short description and some simulation results for a two-converter system [16], which is used to study the power sharing features of the designed control strategies. Table 1 shows parameters of the system.

In practice, master-slave [17], and democratic [18] current sharing strategies are widely used in paralleled converters systems. The major difference between master-slave and democratic current sharing is that master-slave sharing provides a fixed ratio of current sharing in transient and static regimes of operation, while democratic sharing has a variable ratio of current sharing during transients and a fixed ratio in the static regime of operation. This section shows important details

\begin{tabular}{|c|c|c|}
\hline \multicolumn{3}{|c|}{$\begin{array}{c}\text { TABLE } 1 \\
\text { NOMINAL VALUE OF SYSTEM PARAMETERS }\end{array}$} \\
\hline Name & Parameter & Nominal Value \\
\hline Resistance of inductors & $r_{L 1}=r_{L 2}=r_{L n}$ & $0.2 \mathrm{Ohm}$ \\
\hline Filter inductance & $L_{1}=L_{2}=L_{n}$ & $1.3 \mathrm{mH}$ \\
\hline Filter capacitance & $C_{1}=C_{2}=C_{n}$ & $2500 \mu \mathrm{F}$ \\
\hline Input voltage & $E$ & $850 \mathrm{~V}$ \\
\hline Output voltage & $V_{C, r e f}=U_{0}$ & $750 \mathrm{~V}$ \\
\hline \multicolumn{3}{|l|}{ Load uncertainty } \\
\hline Load resistance & $R_{e x t}$ & 2.81-50 Ohm \\
\hline Load capacitance & $C_{L}$ & $0-4900 \mu \mathrm{F}$ \\
\hline Constant power load & $P_{L}$ & $0-200 \mathrm{~kW}$ \\
\hline \multicolumn{3}{|l|}{ Control Law param. } \\
\hline Time constants & $T_{1}=T_{2}=T_{n}$ & 0.0001 \\
\hline Integrator coef. & $\eta=\eta_{n}$ & 50 \\
\hline Law coef. & $a_{11}=a_{11 n}$ & 1 \\
\hline Law coef. & $a_{12}=a_{12 n}$ & 1 \\
\hline Law coef. & $a_{21}=a_{2 \ln }$ & 1 \\
\hline Law coef. & $a_{22}=a_{22 n}$ & -1 \\
\hline Law coef. & $b_{12}=b_{12 n}$ & 1 \\
\hline Law coef. & $b_{11}=b_{11 n}$ & 5 \\
\hline Law coef. & $b_{22}=b_{22 n}$ & 0 \\
\hline Law coef. & $b_{21}=b_{21 n}$ & 0 \\
\hline Law coef. & $\Delta$ & 1 \\
\hline Simulation parameters & Fig3 & \\
\hline Load resistance & $R_{\text {ext }}$ & $10 \mathrm{Ohm}$ \\
\hline Initial current of $L 1$ & $i_{1}(0)$ & $15 \mathrm{~A}$ \\
\hline Initial current of L2 & $i_{2}(0)$ & $0 \mathrm{~A}$ \\
\hline Initial bus voltage & $v_{b}(0)$ & $736 \mathrm{~V}$ \\
\hline Load capacitance & $C_{L}$ & $0 \mu \mathrm{F}$ \\
\hline Constant power load & $P_{L}$ & $0 \mathrm{~kW}$ \\
\hline
\end{tabular}
of the impact of macro-variable coefficients on current sharing in the system and illustrates that synergetic control laws provide power systems with dynamic management of responsibilities, including the dynamic reconfiguration and the allocation of current sharing.

After the transients are passed, the representing point of the two-converter system inevitably reaches the intersection of the manifolds (18). Hence, the motion of the system on the manifolds is defined by the following equations:

$$
\left(\begin{array}{ll}
a_{11} & a_{12} \\
a_{21} & a_{21}
\end{array}\right) \cdot\left(\begin{array}{l}
i_{L 1} \\
i_{L 1}
\end{array}\right)=\left(\begin{array}{l}
b_{11} M+b_{12} v+\frac{b_{14}}{\left(v+V_{c 1, \text { ref }}\right)} \\
\left.b_{21} M+b_{22} v+\frac{b_{24}}{\left(v+V_{c 1, \text { ref }}\right.}\right)
\end{array}\right)
$$

The study shows that:

- coefficients $a_{11}, a_{12}, a_{21}$, and $a_{22}$ allocate the current sharing ratings,

- coefficients $b_{14}$, and $b_{24}$ provide the ability to compensate the impact of the constant power load,

- coefficient $b_{22}$ defines the type of current sharing $\left(\mathrm{b}_{22}=0\right.$ master-slave and democratic otherwise),

- coefficients $b_{11}$ and $b_{12}$ influence the system's transient response,

- time constants $T_{1}$ and $T_{2}$ the define speed of convergence of the macro-variables toward manifolds,

- coefficients $b_{12}$ and $b_{22}$ define which converter is responsible for managing errors in the output voltage. 
Fig.4 presents a state portrait of the two-converter system under synergetic control (22). It can be seen from the figure that representing point of the system goes from initial point $\mathrm{X}(0)$ along the red line onto the intersection of invariant manifolds $\Psi_{1}$ and $\Psi_{2}$ and along the intersection to the equilibrium point $\mathrm{Xe}$.

Based on the simulation results presented in Fig 5., it can be said how the two-converter system implements the defined invariant based power sharing. These invariants are specified in form of algebraic equations (6), coefficients which can compensate the impact of constant power load as well as define properties of current sharing and which can be adjusted dynamically.

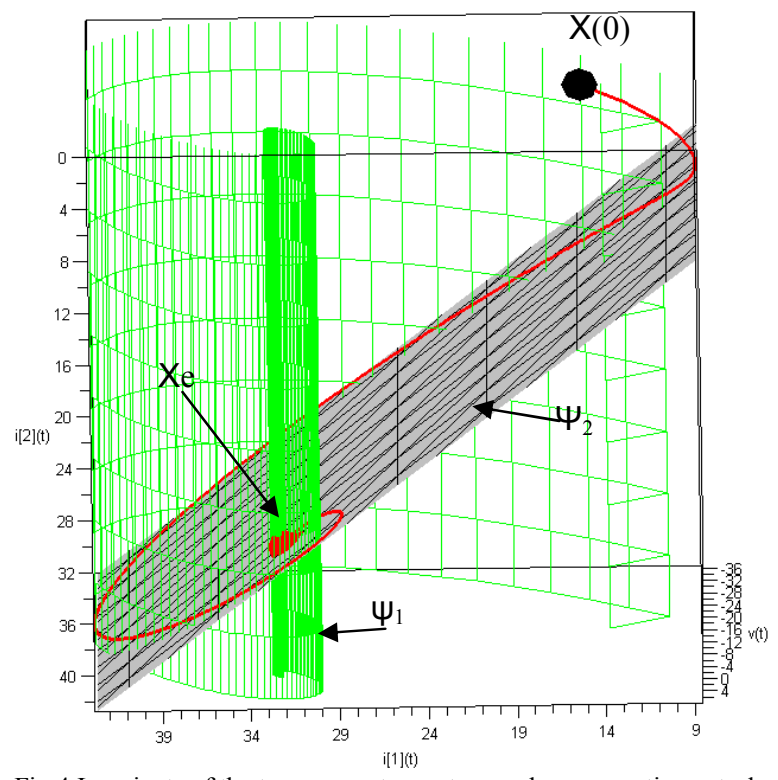

Fig.4 Invariants of the two-converter system under synergetic control

\section{CONCLUSION}

In this paper, we introduce the principle of design of complex dynamic power systems, where attractors and invariants set the rules and define the driving forces of the behavior of system components. This principle has been inspired by examples of similar behaviors in nature, i.e in complex ecological systems. We introduce similar principles in the design of a DC distribution system by providing the system with rules of interaction among its components (invariants) and use computational resources available in the controller to implement such conditions for the system components that they are forced to self-organize the implementation of these rules.

We obtain the rules of in-system interaction by analyzing power sharing in m-paralleled connected power sources feeding a complex load. We illustrate the design using an example of a system with an arbitrary number of paralleled buck converters feeding a constant power load. We introduce the found invariants into the system using synergetic control theory, analyze closed loop performance, and define the stability conditions as well as conditions for compensating impact of constant power load.

The performed simulation of a two-converter system provided evidence that the closed loop system possesses invariant introduced during the control design. More importantly, the simulation proves that the presented concept as well as the design approach provide a foundation for the design of a new type of systems by implementing selforganizing behavior and predefined alternatives for dynamic behavior.

\section{ACKNOWLEDGMENT}

The authors acknowledge the support of ONR grant N00014-08-0080.

\section{REFERENCES}

[1] J.G. Ciezki and R.W. Ashton, "Selection and Stability issues associated with a navy shipboard DC zonal electric distribution system" IEEE Trans on Power Delivery, Vol. 15, no.2, April 2000, pp. 665-669.

[2] S.D.Pekarek and al. "Overview of a Naval Combat Survivability Program", Proceedings of the 13th Ship Control Systems Symposium (SCSS 2003), Orlando, Florida, April 7-9, 2003

[3] S. D. Sudhoff, D.H. Schmucker, R.A. Youngs, and H.J. Hegner, "Stability analysis of DC distribution system using admittance space constraints", SAE International, 04 1998, pp.21-35.

[4] A. Sanchez-Squella, R Ortega, R. Grino, and S. Malo, "Dynamic Energy Router", IEEE Control Systems Magazine, Dec., 2010, pp72-79.

[5] I. Kondratiev and R. Dougal "Synergetic Control Strategies for Shipboard DC Power Distribution Systems" American Control Conference 2007, NY

[6] I. Kondratiev, E.Santi, and R. Dougal "Robust Nonlinear Synergetic Control for m-Parallel-Connected DC-DC Boost Converters" Proc. of IEEE PESC'08, 15-19 June, Rhodes Greece, pp.2222-2228

[7] S. K. Mazunder, A. H. Nayfeh, and D. Borojevic, "Robust Control of Parallel DC-DC Buck Converters by Combining Integral-VariableStructure and Multiple-Sliding-Surfaces Control Schemes," IEEE Trans. on Power Electron, vol. 17, pp.428-437, May 2002.

[8] I. Kondratiev, R. Dougal and G. Veselov "Anti-Chaotic Control for Power Converters Using Synergetic Control Theory" in Proc. Of Chaotic Modeling and Simulation Conference, 3-6 June Chania Crete Greece, 2008, http://www.asmda.net/chaos2008/

[9] Kolesnikov, Synergetic Control Theory. - Energoatomizdat, Moscow, 1994.

[10] Kolesnikov et. al. Modern applied theory of control: synergetics approach in control theory (part II). TSURE publ. / Integracia, Moscow-Taganrog, 2000.

[11] Kondratiev, Synergetic Control: Converter Based Autonomous DC Power Distribution Systems, Lambert Academic Publishing AG \& Co. $\mathrm{KG}, 2009$.

[12] Landau Lifshitz, Statistical Physics Pergamon Press, Oxford, 1980

[13] Kondratiev Geometrical Thynsesis Theory Of Optimal Stationary Smooth Control Systems Fizmatlit, 2003

[14] R. Ericson, S. Cuk, and R. Middlebrook, "Large-scale modeling and analysis of switching regulators," IEEE PESC Rec., 1982, pp. 240-250.

[15] A.A. Kolesnikov, Synergetic Control Theory, Moscow - Taganrog: TSURE, 1994

[16] J. G. Ciezki and R. W. Ashton ,'The Design of Stabilizing Controls for Shipboard DC-to-DC Buck Choppers Using Feedback Linearization Techniques," Proceed. Of PESC'98, pp.335-341

[17] V.J Throttuvelil, and G.C. Verghese, "Analysis and Control Design of Paralleled DC/DC Converters with Current Sharing", IEEE Trans. on Power Electron., Vol. 13, pp.635-644, 1998.

[18] M.M. Jovanovic, D. E. Crow, and L. Fnag-Yi, "A Novel, Low-Cost Implementation of "Democratic" Load-Current Sharing of Paralleled Converter Modules", IEEE Trans. on Power Electron. Vol.11, pp.604$611,1996$. 